\title{
ON THE MODELLING OF COMPLEX SOCIOPSYCHOLOGICAL SYSTEMS WITH SOME REASONING ABOUT KATE, JULES, AND JIM
}

\author{
NICOLA BELLOMO AND BRUNO CARBONARO
}

Received 10 June 2005; Revised 2 November 2005; Accepted 18 January 2006

This paper deals with the modelling of complex sociopsychological games and reciprocal feelings involving interacting individuals. The modelling is based on suitable developments of the methods of mathematical kinetic theory of active particles with special attention to modelling multiple interactions. A first approach to complexity analysis is proposed referring to both computational and modelling aspects.

Copyright (C) 2006 N. Bellomo and B. Carbonaro. This is an open access article distributed under the Creative Commons Attribution License, which permits unrestricted use, distribution, and reproduction in any medium, provided the original work is properly cited.

\section{Introduction}

This paper deals with the modelling of complex sociopsychological games and reciprocal feelings based on some conceptual developments of a new class of kinetic equations recently proposed in the literature to model the evolution of large systems of interacting individuals such that their microscopic state is defined not only by mechanical variables, but also by additional variables, describing social and/or biological functions or behaviors.

The guiding lines of the above mathematical approach is the derivation of an evolution equation for the statistical distribution over the microscopic state, which, as a particular case, may be also related to a somehow intelligent, or at least organized, behavior of interacting individuals, which may be called active particles [33]. Interactions modify both the mechanical state (generally position and velocity) and the above introduced internal state; and those related to mechanical variables do not necessarily obey the laws of classical mechanics, considering that these may turn out to be themselves modified by what we have called an organized behavior.

Specifically, we refer to [2] for the modelling of large systems of entities undergoing short-range interactions, while systems with long-range interactions are dealt with in [7], further developed in [12]. The kinetic theory approach has been developed starting from 
the paper by Jäger and Segel [25], devoted to modelling the social behavior of interacting populations of insects. This research line, developed after the above pioneer paper, is documented in $[3,4,30]$.

One of the main problems to be tackled consists in dealing with the complexity of modelling the evolution of the sociobiological variable. This aspect appears to be particularly relevant when the model refers to personal feelings $[15,16]$, political ideas $[28,29]$, or social competitions [13, 20-22]. Additional applications refer, among others, to modelling multicellular systems in biology [8, 18], swarm dynamics [14, 31, 34], living fluids [35], or vehicular traffic flows $[9,17,24]$, while the interest of applying the methods of kinetic theory to model large systems is documented in the collection of surveys edited in [10].

One of the complexity problems which have to be taken into account in modelling refers to coupling mechanics and self-organization, which may even be related to individual thinking and psychological attitude. On the other hand, in order to simplify the problem in its most technical features, the mechanical aspect could even be neglected in a first approximation. This is obviously restrictive in principle, and can be accepted only when interactions do not explicitly require a mechanical evolution (just to show an example, we may think of a system consisting of two populations whose evolution is simply to reach conclusions and decisions, and whose interactions-between members of the same population as well as between the two different populations-can be simply described as "debates": in such conditions, it does not matter whether the debates are realized by meeting in a chosen place, or by mail, or by phone calls). In a suitable context, mechanical variables have been and can be replaced by behavioral variables [15].

Dealing with the above topics leads to tackle, in addition to the basic difficulty of any attempt to describe the above kind of systems, also the problem of describing multiple interactions $[1,32]$. Then, while two individuals are interacting, the presence of a third individual can substantially affect also their interactions. This is in fact one of the complexity problems discussed in this paper. In other words, for any triple of individuals, the triple of interactions of each of its elements with the binary interactions between the other two must be considered. The basic idea is indeed that each individual not only interacts with any other individual, but also with any binary interaction between two other individuals. Now, each interaction between two (or more) individuals may be described by a function of their actual state variables, and its results can be expressed by a law linking the value of this function with the evolution in time of these latter; or, as we will see in the next sections, it can be described by a transition probability density from a state to another for each individual involved in the interaction: this is the statistical (or stochastic) description of interactions. Such a probability density completely characterizes the interaction, at least in a given context; accordingly, when we say that three (or more) individuals interact at the same time, we say that each interaction between a pair of them is influenced by the presence of all the others, and this influence is expressed by the fact that the transition probability density is conditional depending on their actual states.

This kind of description may be applied in some way to the story of Kate, Jules, and Jim, in the movie "Jules and Jim," directed by François Truffaut, based on the novel of the same title by Henri-Pierre Roché, and played by Jeanne Moreau (Cathérine), Oskar 
Werner (Jules), and Henri Serre (Jim). Referring to their story, one is naturally led to ask whether a mathematical model could be constructed to describe such a complex, probably unpredictable, system. The first answer should be certainly negative. But still, applied mathematics can try to capture and describe some small subsets (particular aspects) of the system. At least, it is worth trying. One of the advantages is that dealing with the above problem obliges to look again and again to the movie we are talking about. In fact, this paper is dedicated to the director, actors, and technical staff of the movie Jules and Jim. The reader of this paper is warmly invited to avoid the thought that the cold mathematics of this paper may attempt to overlap the smallest part of that beautiful movie, which cannot be forgotten by all those who are fond of high-quality cinema.

To tackle the above problems by the methods of kinetic theory means to develop a statistical mechanical theory for interacting subjects with internal intelligent or at least organized microscopic structure, though this latter should be perhaps understood in a sense rather different from usual. Indeed, as we will see in Section 4, the reference to an internal structure can be enlightened by considering each individual as a whole system (population) of interacting subindividuals: this interpretation allows to draw a detailed description of the internal competition between different psychological drives which in $[15,16]$ has been epitomized by the mathematical concept of self-interaction. A self-interaction of any given individual produces in principle a change of its state, so that any of its possible self-interactions is completely characterized as a correspondence law in the set of all its possible states, which associates to each state (before the self-interaction) a subset of states (after the self-interaction). If this last subset is reduced to a single point, then the result of a self-interaction is deterministic; more in general, we have to consider the result of the self-interaction as a probability density function on the set of all possible states, and to acknowledge that such density depends on the starting state. The statistical derivation of such a density can be performed in the framework of the above interpretation of each individual as a population: this of course leads to a number of technical difficulties (e.g., the definition of the number of subunits as well as of the parameters describing their states). And one of the aims of the present paper is just to contribute at least an initial discussion, along with an attempt of solution, of such kind of difficulties.

After the above preliminaries, we are in a position to outline the contents of this paper, which are organized in four more sections which follow this introduction. Specifically, we have the following.

Section 2 deals with the derivation of a class of mathematical equations to model the above outlined complex systems. The analysis is developed in three steps: the first one will be to introduce the concept of a generalized distribution function over the set of microscopic social states of a large system of interacting individuals (this state includes both the mechanical and the social behaviors). The choice of a description in terms of such a distribution function is related to the need to reduce the complexity induced by the large number of individuals and by multiple interactions, as well as to the need to take into account any random modification of interactions when different subsystems of individuals are considered. The second step deals with the modelling of microscopic interactions, binary and triple interaction schemes. Finally, an evolution equation is derived for the above distribution function starting from models of microscopic interactions. 
Sections 3 and 4 show how the mathematical framework designed in Section 2 can be used to model, respectively, the evolution of social systems and personal feelings between partners. As we will see, suitable technical developments are needed to adjust the mathematical framework offered for the first class of systems to the second one.

Section 5 is devoted to a critical analysis of some complexity problems related to various features of the class of models we are dealing with, and to outline some research perspectives. The complexity analysis refers essentially to computational problems, to modelling aspects, and to the implications of multiple interactions.

\section{Mathematical framework}

As already mentioned in Section 1, this paper deals with modelling large systems of interacting individuals with a somehow organized behavior. The analysis developed in the present section essentially aims at the derivation of a mathematical framework which could be sufficiently general to include a variety of specific models, with special attention to the role of multiple interactions. The analogous problem in the case of binary interactions is discussed in [2].

The contents are developed in three steps, each in one of the subsections which follow. The first one is devoted to the statistical representation, the second one to the modelling of microscopic interactions, and finally the last one deals with the actual derivation of the evolution equations.

The analysis is addressed to systems that are homogeneously distributed in space, and such that social interactions are predominant with respect to mechanical interactions. Considering that this paper aims at studying both collective behaviors of large systems of interacting individuals and personal feelings of a small number of individuals, some technical differences between the mathematical treatment of the two kinds of systems need to be pointed out. Therefore, this section deals with the mathematical framework related to the first class of models, while the generalization to the second class of systems will be dealt with in Section 4 .

2.1. On the statistical representation. Consider a physical system consisting of a large number of interacting individuals that may be subdivided into different interacting populations. The analysis developed in what follows is confined to the particular case in which the number of individuals is constant in time for each population. This case can be shown not to entrain any real loss of generality, at least in the framework of a purely theoretic description: it only leads to a loss of information when (as it happens when dealing with social systems) the variation of the numbers of individuals of the considered populations is just one of the experimental data we want to forecast (e.g., in prey-predator models). On the other hand, as we will point out in details in Section 4, it is specially effective in the modelling of personal feelings. It can be easily shown that in such a context, the actual value of the constant number of individuals is quite immaterial and can be arbitrarily assigned.

Bearing all above in mind, and according to [2], the following definitions are proposed. 
Definition 2.1. The physical variable charged to describe the state of each individual of the system is called microscopic state, which is denoted by the variable $\mathbf{u}$, formally written as follows:

$$
\mathbf{u} \in D_{\mathbf{u}} \subseteq \mathbb{R}^{p} .
$$

The space of the microscopic states is called state space.

It is to be carefully noted that the set of all possible states is implicitly assumed to be the same for all the individuals of the system. This is in fact quite obvious, as can be easily acknowledged from the following definition.

Definition 2.2. The description of the overall state of the system, assumed to be divided into $n$ different populations, labelled with the subscript $i$, is given by the one-individual distribution function

$$
f_{i}:(t, \mathbf{u}) \in \mathbb{R}_{+} \times D_{\mathbf{u}} \longrightarrow f_{i}(t, \mathbf{u}) \in \mathbb{R}_{+},
$$

which will be called generalized distribution function for $i=1, \ldots, n$, and such that $f_{i}(t, \mathbf{u}) d \mathbf{u}$ denotes the number of individuals of the $i$ th population whose state, at time $t$, is in the $p$-dimensional interval $[\mathbf{u}, \mathbf{u}+d \mathbf{u}]$.

Remark 2.3. Pair interactions refer to the test individual interacting with a field individual. Triple interactions refer to the test individual interacting with two field individuals. The distribution function stated in Definition 2.2 refers to the test individual. This means that each population will work as a test individual, for example, Kate, Jules, or Jim, or as a field individual according to whether we describe the results of interaction on its distribution function or on the distribution function of the individual interacting with it.

Macroscopic overall quantities are obtained as weighted moments of the distribution function. The zeroth-order moment of $f_{i}$ obviously identifies the number of individuals in each population:

$$
n_{i}(t)=\int_{D_{\mathbf{u}}} f_{i}(t, \mathbf{u}) d \mathbf{u}
$$

As expressed by the integral at right-hand side, $n_{i}$ turns out to be in principle a function of time. But the class of models we plan to consider in the sequel is such that $n_{i}$ can be considered constant in time for any $i$ (i.e., for each population): $n_{i}=n_{i 0}=$ const., so that the function $f_{i}(t, \mathbf{u})$ can be regarded as a probability density when divided by $n_{i 0}$ :

$$
\int_{D_{\mathbf{u}}} f_{i}(t, \mathbf{u}) d \mathbf{u}=1, \quad \forall t \geq 0
$$

for all $i=1, \ldots, n$, and where notation $f_{i}$ has not been modified. In other words, for any $A \subseteq D_{\mathbf{u}}$, the integral

$$
P_{i}(A)=\int_{A} f_{i}(t, \mathbf{u}) d \mathbf{u}
$$

gives the probability to find a member of the $i$ th population whose state lies in $A$. 
A comment is now useful: at a first glance, one could feel that the above assumption of constancy in time for the numbers of individuals of all the involved populations should result in a severe loss of generality, in particular if we note that the probabilistic interpretation of function $f_{i}(t, \mathbf{u})$ can be introduced also when $n_{i}=n_{i}(t)$. But our assumption should instead be interpreted in the opposite sense: considering time-dependent numbers of individuals would add no significant information in the framework of the problems we have in mind to discuss, and would only produce a useless complication in calculations. The explicit consideration of functions $n_{i}=n_{i}(t, \mathbf{u})$ is of the greatest relevance in problems about population dynamics as tumor growth or prey-predator interactions.

It could be now of some interest to recall that each probability density $f_{i}(t, \mathbf{u})$ defines a different one-parameter family of vector random variables, or - what is the same-a set of $p$ (one-parameter families of) scalar random variables. Accordingly, we have, for any $t \in \mathbb{R}_{+}, n p$ random variables $U_{i j}$ (the components of the state random variable).

The first-order moments of such random variables (i.e., their expected values) provide, for each $j \in\{1, \ldots, p\}$ and for $i \in\{1,2, \ldots, n\}$, a quantity

$$
A_{i j}=A_{j}\left[f_{i}\right](t)=\int_{D_{\mathbf{u}}} u_{j} f_{i}(t, \mathbf{u}) d \mathbf{u}
$$

which can be called the activation of the $j$-component of state within the $i$-population at the time $t$. From a conceptual viewpoint, the activation can be taken as a (signed) measure of the relevance of the $j$ th state variable for the state of the $i$ th population. This idea of relevance is of course rather hard to interpret when the considered variable is a coordinate in the classical mechanical sense. Quite different is its sense when the $j$ th state variable is a measure of an attitude, a trend, or a power (a feeling towards another person, artistic or scientific ability, economic power, etc.). And analogously to what happens for kinetic variables, it seems quite reasonable to associate to each state variable an energy term.

The second-order moments of variables $U_{i j}$, (the expected values of the random variables $U_{i j}^{2}$ ) provide the activation energy of the $j$-component of state within the $i$-population at time $t$ :

$$
\mathscr{E}_{i j}=\mathscr{E}_{j}\left[f_{i}\right](t, \mathbf{x})=\int_{D_{\mathbf{u}}} u_{j}^{2} f_{i}(t, \mathbf{u}) d \mathbf{u}
$$

where the definition is given of course only by analogy. But it should be carefully noted that the convention to give the above integral the meaning of an energy is not more arbitrary than the definitions of kinetic and potential energy for mechanical variables. What is to be carefully noted is that, a posteriori, such a definition must be endowed with a real sense by the deduction of balance relations analogous to the ones known in the mechanical framework as power and energy theorem or total energy theorem.

2.2. Modelling microscopic interactions. Consider now the problem of designing suitable models of microscopic interactions which occur between two or more individuals of the same population as well as of two or more different populations. In general, two types of interaction schemes can be proposed. 
(i) Localized (pair, triple) interactions occurring between individuals that are sufficiently close to each other, that is, whose mutual distance is lower than a prescribed critical value (individuals are essentially in contact). It is assumed that the surrounding individuals do not influence the interaction, which is considered to be instantaneous in time, and only pair and triple interactions are assumed to be significant.

(ii) Mean-field-long-range-interactions which occur when the individuals are in a certain action domain. In other words, an individual in a certain position $\mathbf{x}$ perceives the action of all individuals localized into a certain volume around $\mathbf{x}$, which one may call action domain. Again only pair and triple interactions are assumed to be significant.

It should be carefully noted that, according to the above definitions, the difference between localized and long-range interactions is reduced to the difference between the distance limits out of which interactions produce no effects at all. Roughly speaking, the limit for localized interactions is infinitesimal, while the limit for long-range interactions is finite. This difference requires to be carefully formalized.

It is also worth noting that the above classification of interactions, which is endowed with an immediate meaning when the state of interacting individuals is described only in terms of space variables (or, more in general, in terms of positions in a metric space), needs some discussion in the case of social, political, or philosophical characters or, in particular, of feelings, since in all these cases to the spatial coordinates is added at least one more coordinate which is a measure of a psychologic (or social or even intellectual) condition (from now on, for the sake of simplicity, this additional condition-which is in fact the very object of study - will be generally identified as a psychologic condition). Accordingly, we can (and probably should) ask whether (a) a distance between psychologic states can be defined; (b) a total distance between spatial and psychologic states can be defined; (c) interactions between individuals should depend on the total distance (and, more specifically, on the distance between reciprocal feelings) or only on the spatial distance.

As a matter of fact, we should probably consider as the most significant interactions those which are capable to change the psychologic state of a test individual and possibly to produce behaviors aiming in turn at changing the psychologic states of another individual, or at producing other desired results: these interactions will then both depend on the psychologic states and modify them. In such interpretation, a particular behavior is perceived in different ways depending on the psychologic state of the individual perceiving them. The touch of one hand on a cheek is perceived as revealing a trend towards love by a lover, but only as an expression of deep friendship by a friend, and also as an improper attempt to enter one's familiarity by a hostile individual.

According to the above remarks, we should probably divide interactions into four groups. First the contact interactions working at a very short distance, but changing effect and, to say so, sign as a properly defined psychologic distance between the reciprocal feelings grows, so that we should consider them in turn divided into two classes, the former containing the psychologic contact interactions, the latter containing the meanpsychologic-field interactions. The same division into two classes must be reproduced for spatial long-range interactions. The four groups of interactions may then be identified by the following four couples of labels: (spatial contact, psychologic contact), (spatial contact, psychologic mean field), (spatial mean field, psychologic contact), (spatial mean 
field, psychologic mean field). These interactions can obviously be multiple interactions, as far as more than two individuals can have physical contacts (being in the same room, say, and exchanging friendly and/or hostile gestures), or being in contact by mail or telephone. Nevertheless, as we will discuss little later, multiple spatial mean-field interactions are somehow hard to describe and to realize (video conferences are nothing more and nothing less than a reproduction of spatial contact interactions): the unavoidable time lag between subsequent pair interactions makes the description in terms of multiple interactions rather unlikely. The best starting point for a discussion of feelings will be to consider only contact interactions. And, as a matter of fact, the class of models dealt with in this paper has been obtained (in the existing literature) in connection with localized interactions.

The analysis developed in what follows is essentially based on localized interactions considering that individuals may have deep psychologic contacts even at finite distances. Still the analysis of mean-field interactions is an interesting perspective for other types of interacting entities, for example, cells of a biological systems (where long-range interactions have been already used [12, 18]), car drivers on vehicles on a road [17], and so forth.

Bearing all the above mentioned in mind, the following models are proposed.

Model 2.4. Localized pair interactions refer to interactions between a test individual with state $\mathbf{u}_{1}$ belonging to the $i$ th population, and a field individual with state $\mathbf{u}_{2}$ belonging to the $j$ th population. Interactions occur at an encounter rate:

$$
\eta_{i j}\left(\mathbf{u}_{1}, \mathbf{u}_{2}\right): D_{\mathbf{u}} \times D_{\mathbf{u}} \longrightarrow \mathbb{R}_{+}
$$

depending on both the states and on the populations to which the two interacting individuals belong. Moreover, the microscopic states of interacting individuals are modified according to the stochastic description delivered by the transition probability density function:

$$
\varphi_{i j}\left(\mathbf{u}_{1}, \mathbf{u}_{2} ; \mathbf{u}\right): D_{\mathbf{u}} \times D_{\mathbf{u}} \times D_{\mathbf{u}} \longrightarrow \mathbb{R}_{+},
$$

which is such that the integral

$$
P_{i j}\left(A \mid \mathbf{u}_{1}, \mathbf{u}_{2}\right)=\int_{A} \varphi_{i j}\left(\mathbf{u}_{1}, \mathbf{u}_{2} ; \mathbf{u}\right) d \mathbf{u}
$$

is the probability that any test individual of $i$ th population, which is in the state $\mathbf{u}_{1}$, falls into a state in the ( $p$-dimensional) subset $A \subseteq D_{\mathbf{u}}$ after a binary interaction with any field individual of $j$ th population being in the state $\mathbf{u}_{2}$.

In connection with the previous discussion about the distinction between localized and mean-field interactions, we submit to the reader's attention two remarks.

(a) We can assume that the state variable $\mathbf{u}$ is a couple $(\mathbf{x}, \mathbf{v})$, where $\mathbf{x}$ is a space variable, describing the position of any individual in the space, and $\mathbf{v}$ is what we could preliminarily identify as a model variable, expressing the relevant property in consideration (political 
ideas, behavioral trends, or feelings towards another individual). Then the above probability is written as

$$
P_{i j}\left(A \mid \mathbf{x}_{1}, \mathbf{v}_{1}, \mathbf{x}_{2}, \mathbf{v}_{2}\right)=\int_{A} \varphi_{i j}\left(\mathbf{x}_{1}, \mathbf{v}_{1}, \mathbf{x}_{2}, \mathbf{v}_{2} ; \mathbf{u}\right) d \mathbf{u} .
$$

(b) The interactions may be spatially localized, or localized with respect to the state variable $\mathbf{v}$, or both. In the first case, when a suitably defined spatial distance (e.g., the Euclidean distance) $d_{\mathbf{x}}\left(\mathbf{x}_{1}, \mathbf{x}_{2}\right)$ satisfies a condition $d_{\mathbf{x}}\left(\mathbf{x}_{1}, \mathbf{x}_{2}\right)>\ell_{\mathbf{x}}$, then

$$
\varphi_{i j}\left(\mathbf{x}_{1}, \mathbf{v}_{1}, \mathbf{x}_{2}, \mathbf{v}_{2} ; \mathbf{u}\right)=\delta\left(\mathbf{u}-\mathbf{u}_{1}\right)
$$

in the second case, (2.12) holds when a suitably defined distance $d_{\mathbf{v}}\left(\mathbf{v}_{1}, \mathbf{v}_{2}\right)$ satisfies a condition $d_{\mathbf{v}}\left(\mathbf{v}_{1}, \mathbf{v}_{2}\right)>\ell_{\mathbf{v}}$; in the third case, when a suitably defined distance $d_{\mathbf{u}}\left(\mathbf{u}_{1}, \mathbf{u}_{2}\right)$ satisfies a condition $d_{\mathbf{u}}\left(\mathbf{u}_{1}, \mathbf{u}_{2}\right)>\ell_{\mathbf{u}}$.

These relations should give a sufficiently clear expression to the conditions under which an interaction produces no effects.

Model 2.5. Localized triple interactions refer to interactions between a test individual with state $\mathbf{u}_{1}$ belonging to the $i$ th population, and two field individuals, with states $\mathbf{u}_{2}$ and $\mathbf{u}_{3}$, respectively, belonging to the $j$ th population and to the $h$ th population, respectively. Interactions occur at an encounter rate

$$
\eta_{i j h}\left(\mathbf{u}_{1}, \mathbf{u}_{2}, \mathbf{u}_{3}\right): D_{\mathbf{u}} \times D_{\mathbf{u}} \times D_{\mathbf{u}} \longrightarrow \mathbb{R}_{+}
$$

depending on both the states and on the populations to which the three interacting individuals belong. Moreover, the microscopic state of the interacting triple is modified according to the description delivered by the transition probability density function:

$$
\varphi_{i j h}\left(\mathbf{u}_{1}, \mathbf{u}_{2}, \mathbf{u}_{3} ; \mathbf{u}\right): D_{\mathbf{u}} \times D_{\mathbf{u}} \times D_{\mathbf{u}} \times D_{\mathbf{u}} \longrightarrow \mathbb{R}_{+},
$$

which is such that

$$
P\left(A \mid \mathbf{u}_{1}, \mathbf{u}_{2}, \mathbf{u}_{3}\right)=\int_{A} \varphi_{i j h}\left(\mathbf{u}_{1}, \mathbf{u}_{2}, \mathbf{u}_{3} ; \mathbf{u}\right) d \mathbf{u}
$$

is the probability that the test individual, being in the state $\mathbf{u}_{1}$ and belonging to the $i$ th population, falls into a state $\mathbf{u}$ of a ( $p$-dimensional) subset $A \subseteq D_{\mathbf{u}}$ after an interaction with two field individuals being in the states $\mathbf{u}_{2}$ and $\mathbf{u}_{3}$, respectively, and belonging to the $j$ th and to the $h$ th population, respectively.

It is quite obvious that the remarks already laid out for pair interactions keep holding for triple interactions, with obvious changes in the formulation of the conditions under which interactions have no effects. For instance, the third condition is replaced by the following one: when $d_{\mathbf{u}}\left(\mathbf{u}_{1}, \mathbf{u}_{2}\right)>\ell_{\mathbf{u}}$ and $d_{\mathbf{u}}\left(\mathbf{u}_{1}, \mathbf{u}_{3}\right)>\ell_{\mathbf{u}}$, then

$$
\varphi_{i j}\left(\mathbf{x}_{1}, \mathbf{v}_{1}, \mathbf{x}_{2}, \mathbf{v}_{2}, \mathbf{x}_{3}, \mathbf{v}_{3} ; \mathbf{u}\right)=\delta\left(\mathbf{u}-\mathbf{u}_{1}\right) .
$$


The transition probability densities $\varphi_{i j}$ and $\varphi_{i j h}$ satisfy, by their very definitions, the following conditions:

$$
\begin{gathered}
\int_{D_{\mathbf{u}}} \varphi_{i j}\left(\mathbf{u}_{1}, \mathbf{u}_{2} ; \mathbf{u}\right) d \mathbf{u}=1, \quad \forall i, j=1, \ldots, n, \forall \mathbf{u}_{1}, \mathbf{u}_{2} \in D_{\mathbf{u}}, \\
\int_{D_{\mathbf{u}}} \varphi_{i j h}\left(\mathbf{u}_{1}, \mathbf{u}_{2}, \mathbf{u}_{3} ; \mathbf{u}\right) d \mathbf{u}=1, \quad \forall i, j, h=1, \ldots, n, \forall \mathbf{u}_{1}, \mathbf{u}_{2}, \mathbf{u}_{3} \in D_{\mathbf{u}} .
\end{gathered}
$$

2.3. Evolution models. The above described microscopic interactions, corresponding to two different types of modelling, generate two different types of evolution equations. The mathematical model corresponding to localized interactions is the following:

$$
\begin{aligned}
\frac{\partial f_{i}}{\partial t}(t, \mathbf{u}) & \\
= & \sum_{j=1}^{n} \int_{D_{\mathbf{u}}^{2}} \eta_{i j}\left(\mathbf{u}_{1}, \mathbf{u}_{2}\right) \varphi_{i j}\left(\mathbf{u}_{1}, \mathbf{u}_{2} ; \mathbf{u}\right) f_{i}\left(t, \mathbf{u}_{1}\right) f_{j}\left(t, \mathbf{u}_{2}\right) d \mathbf{u}_{1} d \mathbf{u}_{2} \\
& -f_{i}(t, \mathbf{u}) \sum_{j=1}^{n} \int_{D_{\mathbf{u}}} \eta_{i j}\left(\mathbf{u}, \mathbf{u}_{2}\right) f_{j}\left(t, \mathbf{u}_{2}\right) d \mathbf{u}_{2} \\
& +\sum_{j=1}^{n} \sum_{h=1}^{n} \int_{D_{\mathbf{u}}^{2}} \eta_{i j h}\left(\mathbf{u}_{1}, \mathbf{u}_{2}, \mathbf{u}_{3}\right) \varphi_{i j h}\left(\mathbf{u}_{1}, \mathbf{u}_{2}, \mathbf{u}_{3} ; \mathbf{u}\right) f_{i}\left(t, \mathbf{u}_{1}\right) f_{j}\left(t, \mathbf{u}_{2}\right) f_{j}\left(t, \mathbf{u}_{3}\right) d \mathbf{u}_{1} d \mathbf{u}_{2} d \mathbf{u}_{3} \\
& -f_{i}(t, \mathbf{u}) \sum_{j=1}^{n} \sum_{h=1}^{n} \int_{D_{\mathbf{u}}^{2}} \eta_{i j h}\left(\mathbf{u}, \mathbf{u}_{2}, \mathbf{u}_{3}\right) f_{j}\left(t, \mathbf{u}_{2}\right) f_{h}\left(t, \mathbf{u}_{3}\right) d \mathbf{u}_{2} d \mathbf{u}_{3} .
\end{aligned}
$$

As already mentioned, an analogous analysis can be developed in the case of models with long-range interactions, which have been proposed for different types of physical systems, for example, multicellular systems [12], see also [18], or traffic flow modelling [9]. The above model only takes into account binary interactions, their generalizations to multiple interactions is certainly an interesting research perspective.

\section{On the modelling of social systems}

The mathematical structure proposed in Section 2 can be applied to model social dynamics for large systems of interacting populations. As already mentioned in Section 1, some papers are already available in the literature. Specifically, we refer to the papers by Lo Schiavo $[28,29]$ concerning the evolution of social states and political ideas. The same type of physical systems are analyzed by Bertotti and Delitala [13] by means of discrete states models. Simulations developed on individual-based models have been proposed by Galam [20-22] with fascinating outputs on the prediction of political competitions.

It is worth mentioning that the above literature is based on short-interaction models, and on binary interactions only. Discrete states models are derived under the assumption that the microscopic state can attain only a finite number of discrete values. The system is then described by a system of ordinary differential, rather than integro-differential, 
equations. On the other hand, models with multiple interactions are not yet developed despite the fact that recent papers suggest to introduce multiple interactions in the theory of games. An additional limitation of the existing literature is the fact that models are confined to the case of scalar microscopic states even when a multidimensional variable is necessary to a relatively more precise description of the microscopic state.

To complete the critical analysis of the existing literature, it is also worth mentioning, with reference to the above quoted paper [13], that it has been shown how the discretization of the microscopic states simplifies the computational complexity and, if compared with continuous models, allows a relatively more refined analysis of existence and stability properties of equilibrium points. An interesting aspect to be considered is indeed that the above discretization should not be regarded as a way to simplify the model, but as a consistent way to represent social classes grouped into a finite number of sets.

On the other hand, discretizing is the only meaningful procedure step when abstract relations must be applied to real measures, since no outcome of any measurement is a real number with infinitely many decimal digits not produced according to a previously assigned rule.

In general, the line which is followed to develop specific models consists of the sequential steps listed below:

(1) assessment of the microscopic variable charged to describe the physical state of individuals composing the large system;

(2) modelling microscopic interactions, namely the encounter rate and the transition probability density corresponding, as indicated in Section 2, to binary and multiple interactions;

(3) derivation, following again Section 2, of the evolution equation and mathematical statement of problems corresponding to the application of the model;

(4) development of a qualitative and computational analysis of the mathematical problems related to item 3 , to obtain suitable information on the evolution of the system in terms of both the moments and the whole distribution function.

The mathematical framework plotted in Section 2 offers a relatively broader environment for modelling social systems than the one which is offered by models available at present in the literature. For instance, if one looks at the contents of [29] on political dynamics, it is reasonable to work with a multidimensional microscopic variable. Namely, not only political collocation, but maybe at least economical rank and level of education are also to be taken into account. Moreover, interactions may not be still limited to pair interactions, for instance between two opposite parties. Triple interactions may be needed to take into account the presence, nowadays unavoidable, of media which filter the dialogue between parties in competition. The need for multiple interactions seems to be clear also in fields different from the ones considered in this paper as it will be discussed in the last section.

The main problem to be properly analyzed, as we will see in the last section, is that the attempt to increase the level of description necessarily affects the level of complexity related to modelling. Waiting for the above announced analysis, we may observe that complexity refers to predictive models which are supposed to be able to describe the evolution of the system in future times. On the other hand, a relatively richer framework is useful 
for explorative models which are such that different assumptions on microscopic interaction design the scenarios of conceivable outputs. Then suitable political and economical actions can be developed to obtain those microscopic interactions which are needed by the output selected within the scenarios offered by the predictions.

Finally, it is worth emphasizing that the need of multiple interactions motivates the attempt to extend the development of the qualitative analysis to the properties of solutions of the class of models we are dealing with. At present, only the result proposed in [1], concerning the existence of equilibrium solutions for models with local multiple interactions, can be found in existing literature.

\section{On the modelling of personal feelings}

This section deals with an analysis, somehow analogous to that of the previous section, related to the modelling of personal feelings. The mathematical structure proposed in Section 2 has already been used to model the evolution of reciprocal feelings of two interacting partners [16]. Additional developments have been proposed in [15].

The idea developed in [16], and technically improved in [15], consists in assuming that encounters between partners modify their reciprocal feelings: attraction and/or hostility. Encounters are interpreted statistically so that their overall amount allows to deal with the two partners as two interacting populations. Although the above approach may be criticized due to the strong correlations related to the above system, still it is an attractive research topic to be accepted as an approximation of physical reality.

The above cited papers deal with binary encounters, while substantial differences have to be taken into account in the case of multiple interactions. Considering that it is somehow difficult to develop a general abstract approach, in addition to the one already given in Section 2.2, some reasonings can be specifically related to the triangle offered by Cathérine, Jules, and Jim with reference to the movie mentioned in Section 1.

We recall that Jules and Jim are two young men living in Paris in the first years of the second decade of XX century. Though the former is Austrian and the latter is French, they are dear friends. They meet Cathérine and are both attracted to her, though Jules shows his psychologic attitude much more clearly than Jim, who seems at first to hide with care his real feelings under the species of a simple friendship. Cathérine seems in turn to became soon very fond of both of them, but perhaps to feel a bit of attraction towards Jim. Nevertheless, she marries Jules, when this last proposes to her the marriage. Little later, the First World War starts, and Jules must go back to Austria with Cathérine, and the two friends find themselves fighting against each other. After the end of the war, the three friends meet again in Paris and try to live and spend some time together as before, but something is severely changed. In the last period of the story, Cathérine falls into a deep crisis which leads her to kill herself together with Jim.

When dealing with a possible model of the psychologic interactions between Cathérine, Jules, and Jim, we then find ourselves describing a system of three populations. Each of them has at each instant a microscopic state, and their states form the total state of the system. For each person of the drama (population), we can consider the case of a microscopic state identified by a scalar variable. Such a state has a different meaning for each type of interaction. 
(i) For the relation between Jules and Jim, $u$ denotes friendship. Essentially positive values of the microscopic state should be taken into account considering that their relation never assumes a substantial hostility although the intensity of the friendship occasionally decays to zero. However, a distribution over the whole real line will be dealt with, considering that in any relation also negative aspects are always present.

(ii) The variable $u$ denotes attraction right from the beginning for both relations between Cathérine and Jim, and between Cathérine and Jules. Therefore, one should again assume only positive values of the microscopic variable.

However, it should be argued whether a scalar variable is sufficient to identify the emotional state. In other words, one may pose the following question: can a friendship exist without attraction? Apparently, not in the case of this movie. Therefore a description delivered by a scalar variable is simply to be regarded as an attempt to reduce the complexity of the system we are dealing with. Moreover, we are not naive enough to think that such a complex story can be constrained into a mathematical model. The story even includes the First World War separating Jules and Jim, and later the onset of nazism at the same time of the crisis of Cathérine.

Nevertheless we deem that some reasonings about reciprocal feelings of Julies, Jim, and Cathérine can be developed with reference to the first part of the movie, that is, until the marriage of Cathérine and Jules before the war. As an outcome of these reasonings, we will mainly analyze the relevance of the role that multiple interactions can play in this type of modelling.

Before developing the above outlined analysis, it is necessary to show how the mathematical framework offered in Section 2 needs to be technically modified to deal with this specific class of systems. Essentially, one has to identify precisely the variable describing the microscopic state, and subsequently the distribution functions which may describe the above complex relationships. Finally, the framework of Section 2 can be developed towards the mathematical description.

The microscopic states should refer to all specific interchanges of feelings. Each person, identified as a population, is a carrier of three feelings identified by a subscript (related to the carrier) and a superscript (related to the object of the feeling). This entrains that the whole microscopic state must be represented by a matrix

$$
\mathbf{U}=\left(\begin{array}{lll}
u_{1}^{1} & u_{1}^{2} & u_{1}^{3} \\
u_{2}^{1} & u_{2}^{2} & u_{2}^{3} \\
u_{3}^{1} & u_{3}^{2} & u_{3}^{3}
\end{array}\right)
$$

with obvious meaning of terms $u_{j}^{i}$. In particular, for any $i, u_{i}^{i}$ is a measure of self-feelings of $i$ th person that are taken here as needing to be explicitly considered, at least for the sake of completeness.

Remark 4.1. Of course, since we have no reason to guess that self-feelings of Jules, Jim, and Cathérine undergo significant changes, at least before the war, we could assume that for any $i \in\{1,2,3\}, u_{i}^{i}$ is constant on a whole time interval. This is however a very minor remark at this stage. 
Of course, each feeling is a random variable, and we agree to denote by $f_{j}^{i}$ the probability density function associated with $u_{j}^{i}$. On the other hand, another obvious way to write the matrix $\mathbf{U}$ is

$$
\mathbf{U}=\left(\begin{array}{l}
\mathbf{u}_{1} \\
\mathbf{u}_{2} \\
\mathbf{u}_{3}
\end{array}\right),
$$

which has the advantage of pointing out, for each interacting person, the whole complex of its feelings towards itself and towards the other two. Thus, we can express the state variables in vector form, as in the general scheme illustrated in Section 2 (and in particular in the form given by system (2.18)), as well as in the matrix and in the scalar form. To exploit the technical connections between these descriptions, we need to define the probability distributions over the states. To this aim, let us start with assuming a time-dependent probability density function $F:(\mathbf{U}, t) \in \mathbb{R}^{9} \times \mathbb{R} \rightarrow F(\mathbf{U}, t) \in[0,+\infty)$ to be defined. Accordingly, if $R$ is any nine-dimensional rectangle, that is, a subset of $\mathbb{R}^{9}$ defined by constraints

$$
a_{i} \leq x_{i} \leq b_{i}, \quad \forall i \in\{1,2, \ldots, 9\}
$$

then

$$
P(\mathbf{U} \in R, t)=\int_{R} F(\mathbf{U}, t) d \mathbf{U}
$$

is the probability that the matrix variable $\mathbf{U}$ has a value in $R$ at instant $t$. This is obviously a joint probability density, which is to be written in general in the form

$$
F(\mathbf{U}, t)=f_{3}\left(\mathbf{u}_{3}, t \mid \mathbf{u}_{1}, \mathbf{u}_{2}\right) f_{2}\left(\mathbf{u}_{2}, t \mid \mathbf{u}_{1}\right) f_{1}\left(\mathbf{u}_{1}, t\right),
$$

where $f_{i}$ is always a probability density on $\mathbb{R}^{3}$ (i.e., on the set of values of the corresponding row-vector variable $\mathbf{u}_{i}$ ), which is conditioned to the values of variables on the right of vertical line (when there are). If we assume that the feelings of Jules, Jim, and Cathérine are statistically independent, then

$$
F(\mathbf{U}, t)=f_{3}\left(\mathbf{u}_{3}, t\right) f_{2}\left(\mathbf{u}_{2}, t\right) f_{1}\left(\mathbf{u}_{1}, t\right) .
$$

This assumption is acknowledged to be quite acceptable, and seems to fit real situations very well: it is hard to claim that my love towards a friend can depend on whether he loves or hates a woman I love. It can be reinforced or seriously quaked by an interaction which makes me know about his feelings, but still, it is my behavior which is changed by such interaction: I could keep loving my friend even when I should feel to be compelled to stop meeting him.

This established, we note again that also each $f_{i}$ is a joint probability density, which, under the simplified assumption of factorization, writes

$$
f_{i}\left(\mathbf{u}_{i}, t\right)=f_{i}^{3}\left(u_{i}^{3}, t \mid u_{i}^{1}, u_{i}^{2}\right) f_{i}^{2}\left(u_{i}^{2}, t \mid u_{i}^{1}\right) f_{i}^{1}\left(u_{i}^{1}, t\right)
$$


But we feel allowed to repeat almost word by word the above reasoning about the mutual statistical independence of feelings of a person toward different individuals: just to consider an example, there is no reason why the knowledge of my feelings towards my mother, say, should modify an external observer's estimate of probabilities of different levels of my feelings towards, say, my father. Accordingly, we are led to assume that

$$
f_{i}\left(\mathbf{u}_{i}, t\right)=f_{i}^{3}\left(u_{i}^{3}, t\right) f_{i}^{2}\left(u_{i}^{2}, t\right) f_{i}^{1}\left(u_{i}^{1}, t\right) .
$$

In addition, it is important to note that once relation (4.2) has been assumed, then

$$
\int_{-\infty}^{+\infty} d u_{i}^{j} \int_{-\infty}^{+\infty} \frac{\partial f_{i}\left(\mathbf{u}_{i}, t\right)}{\partial t} d u_{i}^{k}=\frac{\partial}{\partial t} \int_{-\infty}^{+\infty} d u_{i}^{j} \int_{-\infty}^{+\infty} f_{i}\left(\mathbf{u}_{i}, t\right) d u_{i}^{k}=\left[\frac{\partial f_{i}^{h}}{\partial t}\right]_{\substack{h \neq j \\ h \neq k}}
$$

Remark 4.2. As a matter of fact, it is possible to show-but, for the sake of simplicity, we may give up this task as immaterial in our context-that relation (4.9) holds even if statistical independence of feelings is not assumed.

When using the scalar representation, and in view of Remark 4.1, we need a system of six evolution equations. For the sake of simplicity, we agree to use instead the vector notation and write down three scalar evolution equations dealing with the three densities:

$$
f_{h}:\left(t, \mathbf{u}_{h}\right) \in \mathbb{R} \times \mathbb{R}^{3} \longrightarrow f_{h}\left(t, \mathbf{u}_{h}\right) \in \mathbb{R}_{+},
$$

rather than with the six densities:

$$
f_{h}^{b}:\left(t, u_{h}^{b}\right) \in \mathbb{R} \times \mathbb{R} \longrightarrow f_{h}^{b}\left(t, u_{h}^{b}\right) \in \mathbb{R}_{+} \quad(h \neq b) .
$$

The evolution system is then as follows:

$$
\begin{aligned}
\frac{\partial f_{h}}{\partial t}\left(t, \mathbf{v}_{h}\right)= & \sum_{i=1}^{3} \iint_{\mathbb{R}^{3}} \eta_{i h}\left(\mathbf{u}_{i}, \mathbf{u}_{h}\right) \varphi_{i h}\left(\mathbf{u}_{i}, \mathbf{u}_{h} ; \mathbf{v}_{h}\right) f_{i}\left(t, \mathbf{u}_{i}\right) f_{h}\left(t, \mathbf{u}_{h}\right) d \mathbf{u}_{i} d \mathbf{u}_{h} \\
& -f_{h}\left(t, \mathbf{v}_{h}\right) \sum_{i=1}^{3} \iint_{\mathbb{R}^{3}} \eta_{i h}\left(\mathbf{u}_{i}, \mathbf{v}_{h}\right) f_{i}\left(t, \mathbf{u}_{i}\right) d \mathbf{u}_{i} \\
& +\iint_{\mathbb{R}^{3}} \eta\left(\mathbf{u}_{1}, \mathbf{u}_{2}, \mathbf{u}_{3}\right) \varphi_{h}\left(\mathbf{u}_{1}, \mathbf{u}_{2}, \mathbf{u}_{3} ; \mathbf{v}_{h}\right) \\
& \times f_{1}\left(t, \mathbf{u}_{1}\right) f_{2}\left(t, \mathbf{u}_{2}\right) f_{3}\left(t, \mathbf{u}_{3}\right) d \mathbf{u}_{1} d \mathbf{u}_{2} d \mathbf{u}_{3} \\
& -f_{h}\left(t, \mathbf{v}_{h}\right) \sum_{i=1}^{3} \sum_{k \neq i} \iint_{\mathbb{R}^{3}} \eta^{*} f_{i}\left(t, \mathbf{u}_{i}\right) f_{k}\left(t, \mathbf{u}_{k}\right) d \mathbf{u}_{i} d \mathbf{u}_{k},
\end{aligned}
$$

where

(1) $\eta^{*} \equiv \eta\left(\left(1-\delta_{1}^{h}\right) \mathbf{u}_{1}+\delta_{1}^{h} \mathbf{v}_{h},\left(1-\delta_{2}^{h}\right) \mathbf{u}_{2}+\delta_{2}^{h} \mathbf{v}_{h},\left(1-\delta_{3}^{h}\right) \mathbf{u}_{3}+\delta_{3}^{h} \mathbf{v}_{h}\right)$

(2) for any couple of indexes $i$ and $j, \delta_{j}^{i}$ is the classical Kronecker's symbol;

(3) we have adopted the convention to denote by $\mathbf{u}_{h}$ the state of $h$ th person before an interaction in the gain term (in which $\mathbf{v}_{h}$ must denote the state after the interaction), and after the interaction in the loss term (where $\mathbf{v}_{h}$ must be the state before the interaction); 
(4) since in the third integral the transition probability density is conditioned by the states of all the persons of the system, simultaneously interacting, we have deemed it right to affect it by only one index;

(5) the reason of the above choice is the same for which the rate of triple encounters is not affected by indexes, there is only one kind of triple interactions;

(6) finally, as it is quite obvious, $d \mathbf{u}_{i} \equiv d u_{i}^{1} d u_{i}^{2} d u_{i}^{3}$ for any $i \in\{1,2,3\}$.

Remark 4.3. If self-feelings were acknowledged to influence the reciprocal feelings, then the present scheme should be considered a simple approximation confined to the period before the war. Nevertheless, the correlation of feelings is a matter of discussion.

The above formulation of the evolution system, together with relation (4.9), shows that one should choose the vector or the scalar representation according to the way in which the transition probabilities $\varphi_{i h}$ and $\varphi_{i h k}$ are defined. More precisely, let us recall that

(i) $\varphi\left(\mathbf{u}_{i}, \mathbf{u}_{h} ; \mathbf{v}_{h}\right)$ is the probability (density) that the state of the $h$ th person is turned from $\mathbf{u}_{h}$ to $\mathbf{v}_{h}$ provided that

(a) while being in the state $\mathbf{u}_{h}$, it had an interaction with the $i$ th person,

(b) at the moment of interaction, the $i$ th person was in the state $\mathbf{u}_{i}$.

Accordingly, its nature of a joint probability allows us to write

$$
\varphi_{i h}\left(\mathbf{u}_{i}, \mathbf{u}_{h} ; \mathbf{v}_{h}\right)=\varphi_{i h}^{p r s}\left(\mathbf{u}_{i}, \mathbf{u}_{h} ; v_{h}^{p} \mid v_{h}^{r}, v_{h}^{s}\right) \varphi_{i h}^{r s}\left(\mathbf{u}_{i}, \mathbf{u}_{h} ; v_{h}^{r} \mid v_{h}^{s}\right) \varphi_{i h}^{s}\left(\mathbf{u}_{i}, \mathbf{u}_{h} ; v_{h}^{s}\right),
$$

where

(i) the third factor at the right-hand side is the probability (density) that the state $\mathbf{u}_{h}$ is transformed by the interaction in any state having $v_{h}^{s}$ as sth component;

(ii) the second factor at the right-hand side is the probability (density) that the state $\mathbf{u}_{h}$ is transformed by the interaction in any state having $v_{h}^{r}$ as $r$ th component, provided that its sth component is $v_{h}^{s}$;

(iii) the first factor at the right-hand side is the probability (density) that the state $\mathbf{u}_{h}$ is transformed by the interaction in any state having $v_{h}^{p}$ as $p$ th component, provided that its $r$ th component is $v_{h}^{r}$ and its sth component is $v_{h}^{s}$.

As it is well-known, (p.r.s) can be an arbitrary permutation of $(1,2,3)$, so that we are allowed to conclude that for any $s \in\{1,2,3\}$

$$
\varphi_{i h}^{s}\left(\mathbf{u}_{i}, \mathbf{u}_{h} ; v_{h}^{s}\right)=\int_{-\infty}^{+\infty} d v_{h}^{p} \int_{-\infty}^{+\infty} \varphi_{i h}\left(\mathbf{u}_{i}, \mathbf{u}_{h} ; \mathbf{v}_{h}\right) d v_{h}^{r} \quad(p \neq s, r \neq s),
$$

obviously, the above argument may be repeated word by word for the transition probability density associated with triple interactions, so that we can also write

$$
\varphi_{h}^{s}\left(\mathbf{u}_{1}, \mathbf{u}_{2}, \mathbf{u}_{3} ; v_{h}^{s}\right)=\int_{-\infty}^{+\infty} d v_{h}^{p} \int_{-\infty}^{+\infty} \varphi_{h}\left(\mathbf{u}_{1}, \mathbf{u}_{2}, \mathbf{u}_{3} ; \mathbf{v}_{h}\right) d v_{h}^{r} \quad(p \neq s, r \neq s) .
$$

Relations (4.14) and (4.15), together with (4.9), show that solving system (4.12) is quite equivalent to solving the system of six equations we would have obtained if we had argued on the densities $f_{h}^{b}$. More precisely, the true criteria to choose as unknown functions the 
three densities $f_{h}$ or the six (or nine) densities $f_{h}^{b}$ are furnished by the way in which the conditional transition probability densities have been assigned (either prescribing the integrals or assigning the functional form of integrands in both (4.14)-(4.15)).

Some reasonings about the identification of the terms $\eta$ and $\varphi$ in the specific case can be now proposed, looking once more at the movie, which goes right from the beginning along this mathematical effort. It is not an easy task, although our analysis will be confined to the first period before the war. Maybe, the feelings are not so extreme as in the second part, but still the intensity induced by new discoveries plays a fascinating role, while it appears impossible to approach, by mathematical equations, an event as a war (that war!) and the consequent separation of human beings.

Bearing all the above in mind, let us identify the persons as follows:

$$
i=1 \text {, Jules, } \quad i=2 \text {, Jim, } \quad i=3 \text {, Cathérine. }
$$

It is plain that the reasonings reported in what follows are derived from a personal interpretation of the authors, while any different interpretation can be expected from different people. So far, some ideas can be put forward, though simply with the aim to open a discussion about this matter.

(i) On the encounter rates The modelling is based on the simplification that the encounter rates do not depend on the personal feelings. Indeed, the three persons have frequent systematic encounters related to their friendships.

(a) The binary encounter rate between Jules and Jim decays in time being replaced by a triple encounter rate:

$$
t \uparrow \Longrightarrow \eta_{12}=\eta_{21} \downarrow
$$

(b) The binary encounter rate between Jules and Cathérine may be described as slowly decaying in time, being (partially) replaced by a triple encounter rate:

$$
t \uparrow \Longrightarrow \eta_{13}=\eta_{31} \downarrow
$$

(c) The binary encounter rate between Jim and Cathérine is negligible, considering that before the war, no personal relation occurs between the two persons: $\eta_{23}=$ $\eta_{32}=0$.

(d) The triple encounter rate reaches very rapidly a constant (in time) value, as soon as Jules introduces Cathérine to Jim.

(e) Relatively more complex is the modelling of the rate of self-interactions related to the intensity of personal feelings. However, a personal opinion of the authors is that Jules examines himself more than the other two on his personal feelings.

The above qualitative indications have not been translated into analytic expressions. The reader interested in some simulations may exploit to this aim simple functions with 
monotonicity properties with respect to their arguments, while condition (4.17) is equivalent to requiring that

$$
\begin{aligned}
& \int_{-\infty}^{+\infty} d v_{1}^{1} \int_{-\infty}^{+\infty} \varphi_{21}\left(\mathbf{u}_{2}, \mathbf{u}_{1} ; \mathbf{v}_{1}\right) d v_{1}^{3}=\delta\left(u_{1}^{2}-v_{1}^{2}\right), \\
& \int_{-\infty}^{+\infty} d v_{1}^{2} \int_{-\infty}^{+\infty} \varphi_{12}\left(\mathbf{u}_{1}, \mathbf{u}_{2} ; \mathbf{v}_{2}\right) d v_{1}^{3}=\delta\left(u_{2}^{1}-v_{2}^{1}\right),
\end{aligned}
$$

as we could easily show bearing in mind the meaning of the encounter rates $\eta_{i h}$. Similar relations hold in the case of other encounters with negligible rates.

Analogous reasonings can be applied to the transition probability densities. For instance, one can think of densities identified by a variance and a most probable value, which may be denoted by the symbol $m_{i h}^{b}$ for each binary encounter and by the symbol $m_{i j h}^{b}$ for each repetition of the triple interaction. The indications which follow simply refer to these variables that now must depend on the microscopic states of interacting persons. The modelling of encounters with zero rate, such as those considered in item (c) of (i), are not taken into account.

(ii) On the binary transition probability densities

(a) Binary encounters between Jules and Jim do not modify their friendship: this is expressed by relations (4.19), which are equivalent to the conditions $\eta_{12}=\eta_{21}=$ 0 , for all $t \geq 0$.

(b) Binary encounters between Jules and Cathérine do not modify the attraction of Jules towards Cathérine:

$$
\int_{-\infty}^{+\infty} d v_{1}^{1} \int_{-\infty}^{+\infty} \varphi_{31}\left(\mathbf{u}_{3}, \mathbf{u}_{1} ; \mathbf{v}_{1}\right) d v_{1}^{2}=\delta\left(u_{1}^{3}-v_{1}^{3}\right)
$$

this is equivalent to stating that $\eta_{13}=\eta_{31}=0$, for all $t \geq 0$, while Cathérine's feelings are modified by triple interactions. Assuming that only these latter are able to modify her feeling means that

$$
\int_{-\infty}^{+\infty} d v_{3}^{2} \int_{-\infty}^{+\infty} \varphi_{13}\left(\mathbf{u}_{1}, \mathbf{u}_{3} ; \mathbf{v}_{3}\right) d v_{3}^{3}=\delta\left(u_{3}^{1}-v_{3}^{1}\right)
$$

Actually, a slow decay of the attraction of Cathérine toward Jules can be observed: this is conveniently expressed by the condition that $m_{3}^{1}$ slowly decays in time.

This delicate aspect is worthy to be discussed before passing to the analysis of triple interactions. Indeed, the feelings of Cathérine towards Jules appear somehow oscillating, at least after she has been introduced to Jim. According to what appears from the movie, she is deeply fond of Jules, but is attracted to Jim. Our guess is that her feelings are modified by triple interactions. 
(iii) On the triple encounters transition probability densities

(a) Triple encounters do not modify the friendship between Jules and Jim:

$$
\begin{aligned}
& \int_{-\infty}^{+\infty} d v_{1}^{1} \int_{-\infty}^{+\infty} \varphi_{1}\left(\mathbf{u}_{1}, \mathbf{u}_{2}, \mathbf{u}_{3} ; \mathbf{v}_{1}\right) d v_{1}^{3}=\delta\left(u_{1}^{2}-v_{1}^{2}\right), \\
& \int_{-\infty}^{+\infty} d v_{2}^{2} \int_{-\infty}^{+\infty} \varphi_{2}\left(\mathbf{u}_{1}, \mathbf{u}_{2}, \mathbf{u}_{3} ; \mathbf{v}_{2}\right) d v_{2}^{3}=\delta\left(u_{2}^{1}-v_{2}^{1}\right) .
\end{aligned}
$$

(b) Triple encounters do not modify the love of Jules for Cathérine:

$$
\int_{-\infty}^{+\infty} d v_{1}^{1} \int_{-\infty}^{+\infty} \varphi_{1}\left(\mathbf{u}_{1}, \mathbf{u}_{2}, \mathbf{u}_{3} ; \mathbf{v}_{1}\right) d v_{1}^{2}=\delta\left(u_{1}^{3}-v_{1}^{3}\right)
$$

On the other hand, these encounters produce an oscillating behavior in the attraction of Cathérine towards Jules. In other words, $m_{312}^{1}$ is oscillating.

(c) Triple encounters increase the attraction of Jim towards Cathérine and vice versa; this is equivalent to stating that $m_{213}^{3}$ and $m_{321}^{2}$ are monotone increasing.

Remark 4.4. There is another way to express the oscillating character of the transition probability density of feelings of Cathérine, in terms of its conditional parameters (expected value and variance as functions of the triple of states at encounters). In any case, the (stochastically) oscillating behavior of the variable $u_{3}^{1}$ may be expressed by assuming the existence of a threshold value $v_{3}^{* 1}$ such that for any $v_{3}^{1}<v_{3}^{* 1}$,

$$
\begin{aligned}
\int_{-\infty}^{v_{3}^{1}} d u_{3}^{1} \int_{-\infty}^{+\infty} d u_{3}^{2} \int_{-\infty}^{+\infty} \varphi_{123}^{1}\left(\mathbf{u}_{3}, \mathbf{u}_{1}, \mathbf{u}_{2} ; v_{3}^{1}\right) d u_{3}^{3} \\
\quad<\int_{v_{3}^{1}}^{+\infty} d u_{3}^{1} \int_{-\infty}^{+\infty} d u_{3}^{2} \int_{-\infty}^{+\infty} \varphi_{123}^{1}\left(\mathbf{u}_{3}, \mathbf{u}_{1}, \mathbf{u}_{2} ; v_{3}^{1}\right) d u_{3}^{3},
\end{aligned}
$$

while, for any $v_{3}^{1}>v_{3}^{* 1}$,

$$
\begin{aligned}
\int_{-\infty}^{v_{3}^{1}} d u_{3}^{1} \int_{-\infty}^{+\infty} d u_{3}^{2} \int_{-\infty}^{+\infty} \varphi_{123}^{1}\left(\mathbf{u}_{3}, \mathbf{u}_{1}, \mathbf{u}_{2} ; v_{3}^{1}\right) d u_{3}^{3} \\
\quad>\int_{v_{3}^{1}}^{+\infty} d u_{3}^{1} \int_{-\infty}^{+\infty} d u_{3}^{2} \int_{-\infty}^{+\infty} \varphi_{123}^{1}\left(\mathbf{u}_{3}, \mathbf{u}_{1}, \mathbf{u}_{2} ; v_{3}^{1}\right) d u_{3}^{3} .
\end{aligned}
$$

Remark 4.5. The very special system above is such that triple encounters play the most relevant role in the evolution (or storm) of feelings, while binary encounters become ever less effective as time increases. Therefore, the asymptotic behavior of the solution can be analyzed by equations involving only triple encounters.

Remark 4.6. The evolution of the system, and hence its asymptotic behavior, is obtained as a solution of the initial value problem. The mathematical structure of the equations is essentially the same as that analyzed in [13]. Therefore, a technically analogous method can be applied to show existence and uniqueness of solutions in the positive cone of a suitable space of integrable functions endowed with an $L_{1}$ norm. 


\section{Complexity analysis}

The preceding sections have shown how certain mathematical structures can be used to model the evolution of both social behaviors and personal feelings. The approach appears to be, in the authors' opinion, quite interesting although the contents of the literature in the field is still far from having proposed robust models. On the other hand, the topics dealt with in this paper are so fascinating that it is worth continuing along this research line, looking forward to relatively more advanced models.

The first step in dealing with the above matter may be a critical analysis related to the specific contents of this paper. So far, bearing in mind various definitions and interpretations of what is called complexity, we will concentrate our attention on the link between complexity problems related to modelling and the computational effort needed to deal with the application of models to the interpretation of physical systems. The existing literature reports various valuable contributions on this topic, for instance, an interesting paper by Vicsek [34]. The following general definitions of complexity are given with reference to living systems.

Collective behavior is: the way in which an individual unit's activity is dominated by its neighbors so that all units simultaneously alter their behavior to a common pattern.

The laws that describe the behavior of a complex system are qualitatively different from those that govern its units.

Let us now be a little more precise on this point and identify by $\mathscr{X}$ the dimension of a model. In more details, $\mathscr{X}$ may be related to the number of equations which are necessary to provide a consistent model. Moreover, let us indicate by 9 a quantity suitable to measure the computational time needed to deal with $\mathscr{X}$-model.

The definition of complexity which is here proposed is the following.

Computational complexity occurs when the growth of $\mathscr{Y}$ with respect to $\mathscr{X}$ is greater than the linear growth. As an extreme case, $\mathscr{Y}$ may grow exponentially with $\mathscr{X}$.

The above definition can be used supposing that models with growing complexity are always available. This is indeed the case of modelling the dynamics of classical particles. The equations of the particle dynamics can be derived on the basis of classical mechanics (Newton) without technical difficulty when the number of particles increases. On the other hand, the computational time increases first linearly and then exponentially when the number of particles and hence of differential equations increases.

The above situation does not apply to all physical systems. For instance, in some cases, increasing the number of variables to describe a certain physical system may increase the difficulty of deriving the mathematical model. Suppose that one is able to provide a measure $\mathscr{E}$ to the above effort, which can be regarded as an "experimental effort," then an additional definition can be given.

Modelling complexity occurs when the growth of experimental effort $\mathscr{E}$ with respect to $\mathscr{X}$ is greater than the linear growth. As an extreme, case $\mathscr{E}$ may grow exponentially with $\mathscr{d}$.

The analysis of the above complexity problems can be properly developed only with reference to specific models validated as reliable and robust. An effort to deal with the above problems will be developed in this section with reference to the applications proposed in Section 3. 
Considering the technical difficulty related to such a problem, only some specific aspects are discussed in details:

(i) complexity problems induced by the selection of the variables suitable to identify the microscopic state;

(ii) complexity problems induced by multiple interactions;

(iii) finalization of the model and selection of the structure of the mathematical equations related to the type of microscopic interactions.

The above topics are treated in the sections which follow. Possibly an answer, however limited, to each of the above topics may give a contribution to improving the validity of the class of models dealt with in this paper.

5.1. On the selection of the microscopic state variable. The microscopic state of interacting individuals is identified, as we have seen, by a variable

$$
\mathbf{u} \in D_{\mathbf{u}} \subseteq \mathbb{R}^{m},
$$

which refers to the specific sociopolitical (or also personal feelings) state of each microscopic entity, the description of the whole system being given by the distribution function

$$
f=f(t, \mathbf{u}):[0, T] \times D_{\mathbf{u}} \longrightarrow \mathbb{R}_{+},
$$

which has to be regarded as the dependent variable.

Consider first models with localized only binary interactions-multiple interactions are considered in the next section. The actual computation of $\mathscr{X}$ can be obtained by reasoning along the discretization scheme proposed in [13], which essentially means discretizing the set $D_{\mathbf{u}}$ into a suitable finite number of states $\mathbf{u}_{i}$ as follows:

$$
I_{\mathbf{u}}=\left\{\mathbf{u}_{1}, \ldots, \mathbf{u}_{i}, \ldots, \mathbf{u}_{n}\right\}
$$

and then deriving an evolution equation for the densities $f_{i}(t)=f_{i}\left(t, \mathbf{u}_{i}\right)$.

The structure of the evolution equation is then, still according to [13], the following system of $p$ ordinary differential equations with quadratic-type nonlinearities:

$$
\frac{d f_{i}}{d t}=\sum_{h=1}^{n} \sum_{k=1}^{n} \eta_{h k} A_{h k}^{i} f_{h} f_{k}-f_{i} \sum_{k=1}^{n} \eta_{i k} f_{k},
$$

for $i=1, \ldots, n$ and where the terms $A_{h k}^{i}$ denote the probability density that an individual with state $h$ falls into the state $i$ due to the encounter with an individual with state $k$. The encounter rates are identified by the terms $\eta_{h k}$ and $\eta_{i k} . A_{h k}^{i}$ has the structure of a discrete probability density:

$$
\forall h, \quad k: \sum_{i=1}^{n} A_{h k}^{i}=1 .
$$

From the above reasoning, one may argue that the measure of $\mathscr{X}$ can be related to $n$. Moreover, if $q$ is the number of collocation points needed to discretize each component 
of $\mathbf{u}$, and $s$ is the number of components of $\mathbf{u}$, then the number of equations is $n=$ $q \times s$. Hence the computational complexity to deal with $q \times s$ equations may soon grow exponentially. Such a complexity may possibly be controlled by selecting, with physical arguments, a lower number $n$ of discrete values.

The above ideas can be used also to analyze the complexity related to modelling. Indeed, the measure of $\mathscr{E}$ can be related to the number of experiments needed to identify the interaction terms. Referring to the encounter rate, one has $n \times n$ terms which include also the self-interactions introduced in [16]. Otherwise, excluding the above artificial selfencounters, their number would be simply $n \times(n-1)$. Similarly for the transition rates, one has $n \times n \times n$ terms. Therefore, the modelling complexity grows more rapidly than the number of equations.

Then we may conclude that a predictive model can be practically handled only if the dimension of the microscopic variable is sufficiently small. However, this matter needs to be discussed further taking into account the effective finalization of the last subsection.

5.2. Dealing with multiple interactions. The reasoning proposed in Section 5.1 can be generalized, with reference to computational complexity, to the case of models with multiple interactions simply by observing that the number of ordinary differential equations is not modified by the presence of multiple interactions. The only technical difference is that each equation is now somehow relatively stiffer.

The structure of the discrete equation can be written as follows:

$$
\frac{d f_{i}}{d t}=\sum_{h=1}^{n} \sum_{k=1}^{n} \sum_{\ell=1}^{n} \eta_{h k \ell} T_{h k \ell}^{i} f_{h} f_{k} f_{\ell}+\sum_{h=1}^{n} \sum_{k=1}^{n} \eta_{h k} A_{h k}^{i} f_{h} f_{k}-f_{i} \sum_{k=1}^{n} \eta_{i k} f_{k}-f_{i} \sum_{k=1}^{n} \sum_{\ell=1}^{n} \eta_{i k \ell} f_{k} f_{\ell},
$$

for $i=1, \ldots, n$, and where the terms $T_{h k \ell}^{i}$ denote the probability density that an individual with state $h$ falls into the state $i$ due to the encounter with two individuals with states $k$ and $\ell$, respectively, while the encounter rates related to binary and triple encounters are identified by the terms $\eta_{h k}$ and $\eta_{h k \ell}$, respectively.

As above, $T_{h k \ell}^{i}$ has the structure of a discrete probability density:

$$
\forall h, \quad k, \ell: \sum_{i=1}^{n} A_{h k \ell}^{i}=1
$$

An additional complexity source, already documented in Section 4, is that the number of variables describing the microscopic state increases with the increasing number of interacting individuals: a square matrix, rather than a string, is needed to identify their state. This type of complexity may be not important in the case of large population where binary interactions play the relevant role, while it may be significant in a small system such as the one analyzed in this paper.

The above reasoning related to computational complexity follows, while the complexity related to modelling is noticeably increased with respect to the models with binary 
interactions only. Still multiple interactions cannot be neglected for some specific physical systems, as discussed in Section 4.

5.3. On the selection of the structure of mathematical equations. This paper has been devoted to a complexity analysis related to a class of sociobiological systems. Actually, the use of the term complexity, or complex systems, can often be recovered in the scientific literature with different meanings related to different objects: physical systems, mathematical models, mathematical problems, and so on. In principle, all real systems are complex in the sense that only an infinite number of variables may possibly capture all the inner features of the model. On the other hand, a mathematical model can capture only a finite number of variables. Therefore, modelling is always a path to reducing the complexity of real world.

Increasing the number of variables should, at least in principle, bring the description of the model closer to physical reality. However, the counterpoint is that increasing the number of variables generally increases the computational time to obtain a careful solution of the mathematical problems related to the application of the model. Referring specifically to the class of problems we are dealing with, one can recognize that complexity is an inner feature of the system itself. In fact, the description of the individuals' behavior definitively needs a large, in principle infinite, number of variables. Moreover, interactions not only increase the number of equations to take into account the number of individuals, but may even need an enlargement of the variables necessary to identify the state of each individual. The above modelling approach has to be regarded as a conceivable way to deal with the complexity of the system and the related models and problems.

The above analysis should be related to the finalization of the model. Indeed, it is worth distinguishing between the following two types of models:

(i) predictive models which should describe the future behavior of the system given suitable initial conditions;

(ii) explorative models which should investigate conceivable future behaviors of the system given suitable initial conditions and samples of microscopic interactions.

In both cases, the computational complexity is the same. On the other hand, we have seen that modelling complexity increases more rapidly than the computational one. This is true in the case of predictive models, while the explorative ones may be used on the basis of suitable a priori assumptions on microscopic interactions. The model is used to outline the scenarios of events, thus identifying (and operating towards) those microscopic interactions which are needed to obtain the desired events.

The selection of the type of evolution equations corresponding to short-range or longrange interactions is related to the actual physics of the system. In some cases, as discussed in Section 4, both types of interactions are consistent, for instance, local interactions for binary encounters and mean-field for triple ones. In the case of explorative models, both types should be investigated.

It is plain that a crucial aspect of complexity is somehow related to the joint presence, in the mathematical model, of mechanistic and organized, possibly even intelligent, behaviors. The term mechanistic behavior refers to the general framework of classical mechanics, say force equilibrium, conservation of mass, momentum, and energy. On the other 
hand, the term organized behavior is used to identify dynamical rules induced by the sociobiological or thinking behaviors of the various individuals belonging to the system which has been put into the framework of a mathematical model.

It is a delicate problem-indeed an interesting research perspective-arising in fields different from the one dealt with in this paper, where interacting individuals modify the dynamics of the system due to their reasoning or organized behavior. In this case, the modelling must go far beyond the description given in this paper, taking advantage of the whole variety of models of the mathematical kinetic theory: an interesting analysis is proposed in [35], where some suggestions to develop new mathematical structures could be found.

Finally, let us mention that a closely related problem is the derivation of macroscopic equations from the microscopic ones by a suitable asymptotic theory. This is a classical topic for models of the mathematical kinetic theory, as documented in various papers (among others [11,27]), while various recent papers have been oriented to derive macroscopic models for active particles $[5,6,19,23,26]$, however confined to binary interactions. It is a challenging mathematical topic, and certainly worthy to be studied at least in the case of the dynamics of populations of active particles.

\section{References}

[1] L. Arlotti and N. Bellomo, Solution of a new class of nonlinear kinetic models of population dynamics, Applied Mathematics Letters 9 (1996), no. 2, 65-70.

[2] L. Arlotti, N. Bellomo, and E. De Angelis, Generalized kinetic (Boltzmann) models: mathematical structures and applications, Mathematical Models \& Methods in Applied Sciences 12 (2002), no. $4,567-591$.

[3] L. Arlotti, N. Bellomo, and M. Lachowicz, Kinetic equations modelling population dynamics, Transport Theory and Statistical Physics 29 (2000), no. 1-2, 125-139.

[4] L. Arlotti, N. Bellomo, and K. Latrach, From the Jager and Segel model to kinetic population dynamics nonlinear evolution problems and applications, Mathematical and Computer Modelling 30 (1999), no. 1-2, 15-40.

[5] N. Bellomo and A. Bellouquid, From a class of kinetic models to the macroscopic equations for multicellular systems in biology, Discrete and Continuous Dynamical Systems. Series B 4 (2004), no. $1,59-80$.

[6] - On the onset of non-linearity for diffusion models of binary mixtures of biological materials by asymptotic analysis, International Journal of Non-Linear Mechanics 41 (2006), no. 2, 281293.

[7] N. Bellomo, A. Bellouquid, and M. Delitala, Mathematical topics on the modelling complex multicellular systems and tumor immune cells competition, Mathematical Models \& Methods in Applied Sciences 14 (2004), no. 11, 1683-1733.

[8] N. Bellomo and E. De Angelis, Strategies of applied mathematics towards an immunomathematical theory on tumors and immune system interactions, Mathematical Models \& Methods in Applied Sciences 8 (1998), no. 8, 1403-1429.

[9] N. Bellomo, M. Delitala, and V. Coscia, On the mathematical theory of vehicular traffic flow. I. Fluid dynamic and kinetic modelling, Mathematical Models \& Methods in Applied Sciences 12 (2002), no. 12, 1801-1843.

[10] N. Bellomo and M. Pulvirenti (eds.), Modeling in Applied Sciences. A Kinetic Theory Approach, Modeling and Simulation in Science, Engineering and Technology, Birkhäuser Boston, Massachusetts, 2000. 
[11] A. Bellouquid, On the asymptotic analysis of kinetic models towards the compressible Euler and acoustic equations, Mathematical Models \& Methods in Applied Sciences 14 (2004), no. 6, 853882.

[12] A. Bellouquid and M. Delitala, Mathematical methods and tools of kinetic theory towards modelling complex biological systems, Mathematical Models \& Methods in Applied Sciences 15 (2005), no. 11, 1639-1666.

[13] M. L. Bertotti and M. Delitala, From discrete kinetic and stochastic game theory to modelling complex systems in applied sciences, Mathematical Models \& Methods in Applied Sciences 14 (2004), no. 7, 1061-1084.

[14] H. Bessaih and F. Flandoli, Limit behaviour of a dense collection of vortex filaments, Mathematical Models \& Methods in Applied Sciences 14 (2004), no. 2, 189-215.

[15] B. Carbonaro and C. Giordano, A second step towards a stochastic mathematical description of human feelings, Mathematical and Computer Modelling 41 (2005), no. 4-5, 587-614.

[16] B. Carbonaro and N. Serra, Towards mathematical models in psychology: a stochastic description of human feelings, Mathematical Models \& Methods in Applied Sciences 12 (2002), no. 10, 14531490.

[17] M. Delitala, Nonlinear models of vehicular traffic flow-new frameworks of the mathematical kinetic theory, Comptes Rendus Mecanique 331 (2003), no. 12, 817-822.

[18] L. Derbel, Analysis of a new model for tumor-immune system competition including long-time scale effects, Mathematical Models \& Methods in Applied Sciences 14 (2004), no. 11, 1657-1681.

[19] F. Filbet, P. Laurençot, and B. Perthame, Derivation of hyperbolic models for chemosensitive movement, Journal of Mathematical Biology 50 (2005), no. 2, 189-207.

[20] S. Galam, Modelling rumors: the no plane Pentagon French hoax case, Physica A: Statistical Mechanics and Its Applications 320 (2003), no. 1-4, 571-580.

[21] _ Contrarian deterministic effects on opinion dynamics: "the hung elections scenario", Physica A: Statistical Mechanics and Its Applications 333 (2004), no. 1-4, 453-460.

[22] _ Sociophysics: a personal testimony, Physica A: Statistical and Theoretical Physics 336 (2004), no. 1-2, 49-55.

[23] K. P. Hadeler, T. Hillen, and F. Lutscher, The Langevin or Kramers approach to biological modeling, Mathematical Models \& Methods in Applied Sciences 14 (2004), no. 10, 1561-1583.

[24] D. Helbing, Traffic and related self-driven many-particle systems, Reviews of Modern Physics 73 (2001), no. 4, 1067-1141.

[25] E. Jäger and L. A. Segel, On the distribution of dominance in populations of social organisms, SIAM Journal on Applied Mathematics 52 (1992), no. 5, 1442-1468.

[26] M. Lachowicz, Micro and meso scales of description corresponding to a model of tissue invasion by solid tumours, Mathematical Models \& Methods in Applied Sciences 15 (2005), no. 11, 16671683.

[27] P.-L. Lions and N. Masmoudi, From the Boltzmann equations to the equations of incompressible fluid mechanics. I, Archive for Rational Mechanics and Analysis 158 (2001), no. 3, 173-193.

[28] M. Lo Schiavo, Population kinetic models for social dynamics: dependence on structural parameters, Computers \& Mathematics with Applications 44 (2002), no. 8-9, 1129-1146.

[29] _ The modelling of political dynamics by generalized kinetic (Boltzmann) models, Mathematical and Computer Modelling 37 (2003), no. 3-4, 261-281.

[30] A. Mogilner and L. Edelstein-Keshet, A non-local model for a swarm, Journal of Mathematical Biology 38 (1999), no. 6, 534-570.

[31] H. G. Othmer, S. R. Dunbar, and W. Alt, Models of dispersal in biological systems, Journal of Mathematical Biology 26 (1988), no. 3, 263-298.

[32] T. Platkowski, Evolution of populations playing mixed multiplayer games, Mathematical and Computer Modelling 39 (2004), no. 9-10, 981-989. 
26 Modelling of sociopsychological systems: Jules and Jim

[33] F. Schweitzer, Brownian Agents and Active Particles. Collective Dynamics in the Natural and Social Sciences, Springer Series in Synergetics, Springer, Berlin, 2003.

[34] T. Vicsek, A question of scale, Nature 411 (2001), no. 6836, 421.

[35] M. Willander, E. Mamontov, and Z. Chiragwandi, Modelling living fluids with the subdivision into the components in terms of probability distributions, Mathematical Models \& Methods in Applied Sciences 14 (2004), no. 10, 1495-1520.

Nicola Bellomo: Department of Mathematics, Politecnico di Torino, 10129 Torino, Italy

E-mail address: nicola.bellomo@polito.it

Bruno Carbonaro: Department of Mathematics, Second University of Napoli, 81100 Caserta, Italy

E-mail address: bruno.carbonaro@unina2.it 


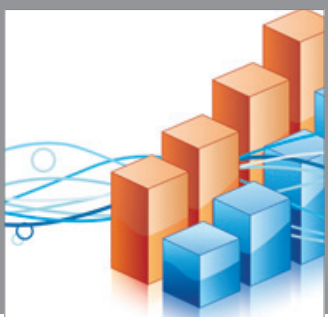

Advances in

Operations Research

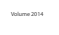

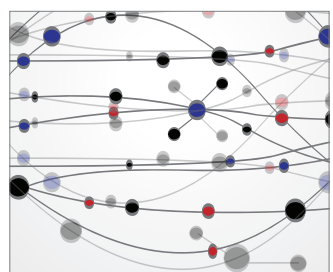

\section{The Scientific} World Journal
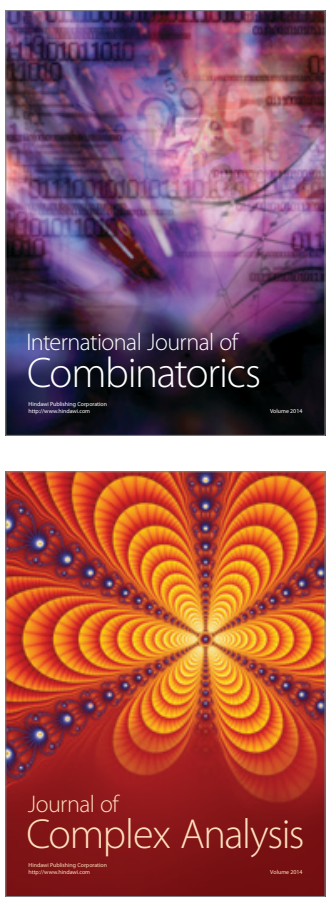

International Journal of

Mathematics and

Mathematical

Sciences
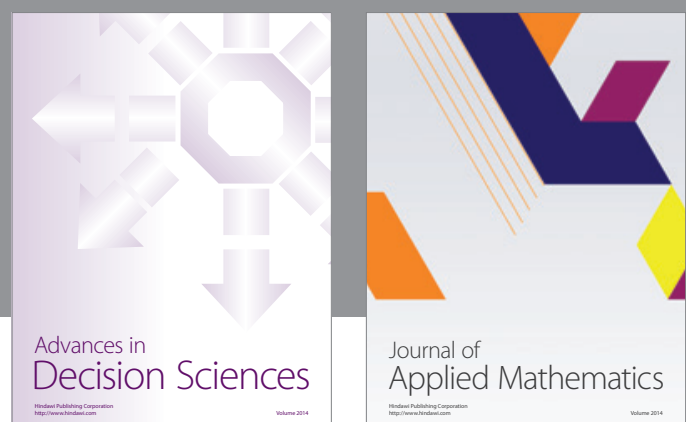

Journal of

Applied Mathematics
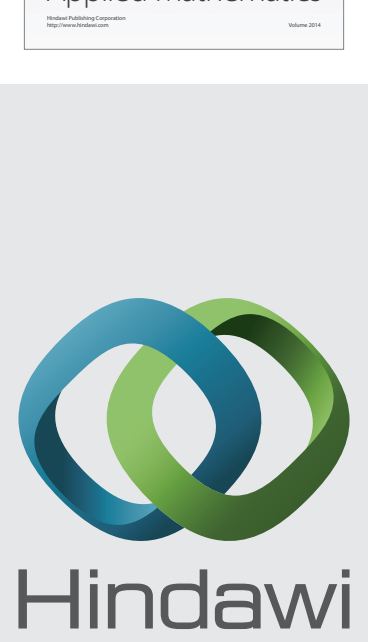

Submit your manuscripts at http://www.hindawi.com
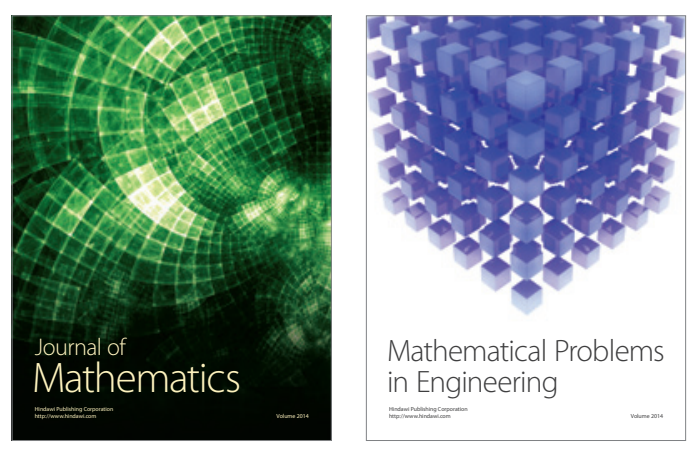

Mathematical Problems in Engineering
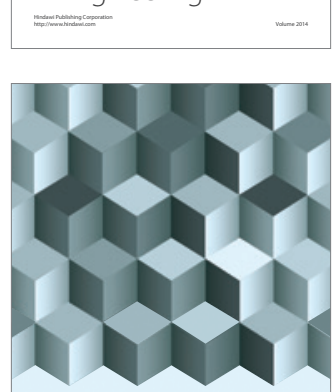

Journal of

Function Spaces
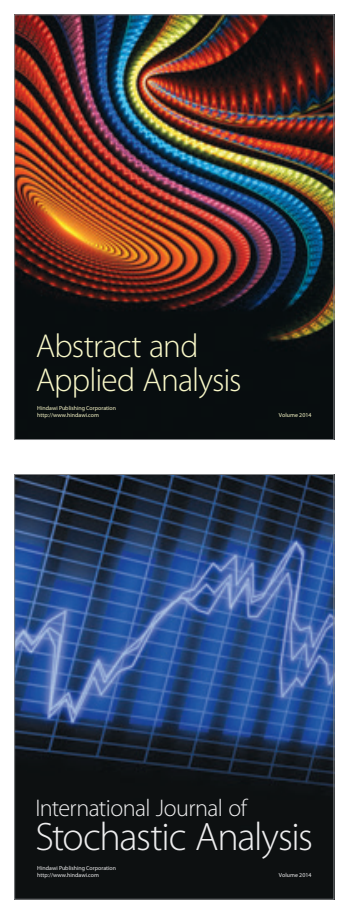

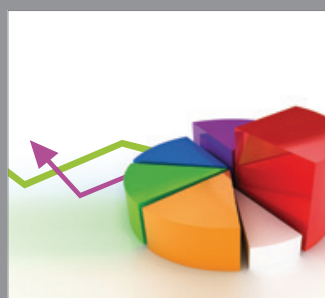

ournal of

Probability and Statistics

Promensencen
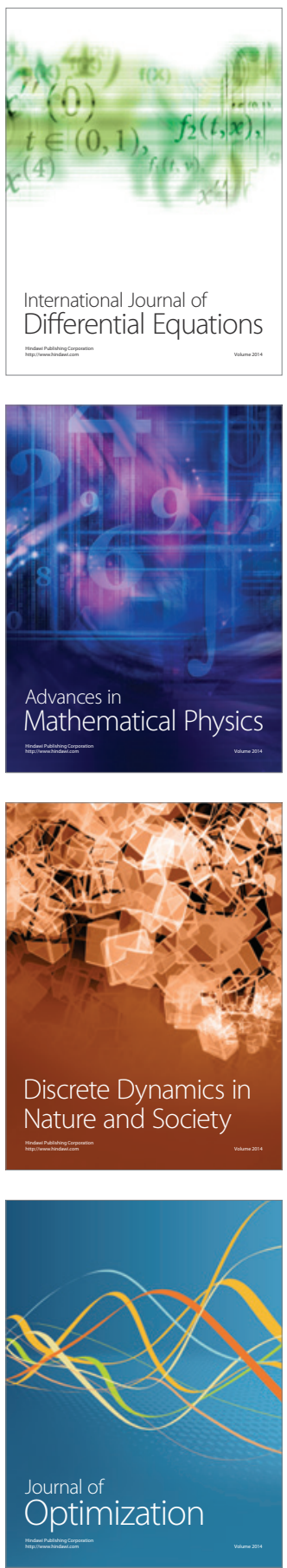